\title{
Domain Ontology of the VirDenT System
}

\author{
C.M. Bogdan
}

\author{
Crenguța Mădălina Bogdan \\ Ovidius University of Constanta, Romania \\ Numerical Methods and Computer Science Department \\ 124 Mamaia Blvd., 900527 \\ E-mail: cbogdan@univ-ovidius.ro
}

\begin{abstract}
The goal of using virtual and augmented reality technologies in therapeutic interventions simulation, in the fixed prosthodontics (VirDenT) project, is to increase the quality of the educational process in dental faculties, by assisting students in learning how to prepare teeth for all-ceramic restorations. Its main component is an e-learning virtual reality-based software system that will be used for the developing skills in grinding teeth, needed in all-ceramic restorations. This paper presents a domain ontology that formally describes the knowledge of the domain problem that the VirDenT e-learning system dealt with. The ontology was developed based on the UML models of the VirDenT information system, making sure in this way the ontology captures knowledge identified and described in the analysis of the information system. At first, we constructed the taxonomy of these concepts, using the DOLCE ontology and its modules. Then, we defined the conceptual relations between the concepts. We also added SWRL rules that formally describe the business rules and knowledge previously identified. Finally, with the assistance of the Pellet reasoner system, we checked the ontology consistency.

Keywords: knowledge, ontology, taxonomy, information system, UML diagram.
\end{abstract}

\section{Introduction}

The goal of using virtual and augmented reality technologies in therapeutic interventions simulation in the fixed prosthodontics (VirDenT) project is to increase the quality of the educational process in the dental faculties, by helping students to learn how to prepare teeth for ceramic crowns.

The VirDenT system is an e-learning system that will be used as a software tool by the students of the dental medicine faculty, to developing their skills in grinding teeth during the dental laboratories of fixed prosthetic department.

These skills are required in making one of the crown types: all-ceramic. All-ceramic restorations are one of the most successfully restorations available from the point of view of aesthetics and biocompatibility. The first criterion is fulfilled by the crown translucent close to the dental hard tissues allowing light to pass through, at gingival level. Biocompatibility refers to the adhesion degree of the ceramic with the surrounding tissue of the teeth. It also refers to how much gingivae tolerate the crown. At present, ceramic appears to be more biocompatible than other dental material [15].

However, this kind of restorations is not widely used because ceramic crowns preparation requires strict and delicate precision. In addition, the bonding techniques employed are somewhat complex and adverse effects could appear, such as pulp inflammation. That is why, the VirDenT system will be a useful tool to the students of dental medicine faculties. 
The first phase of the developing process of the VirDenT software system consisted of the analysis of the information system supported by VirDenT. During the analysis a series of models have been created such context diagram, domain model, business process model, and so on [2]. All these models have been created using the Unified Modelling Language (UML) [9] which is a standard language and allows creating graphic models of the information or software system. The ontology construction method is based on these models and therefore we give further two of the models: domain model and business process model.

Domain model describes structurally the VirDenT information system, i.e. considers only persistent information used by the system. Information is classified in classes together with their relationships which form the UML class diagram of the model domain. The classes emerged from the concepts of the problem domain such as Tooth, Root, Gingiva, Enamel, DiamondTool, and so on (Figure 1). The classes are linked together by generalization/specialization relations or different kinds of associations such as aggregations or compositions.

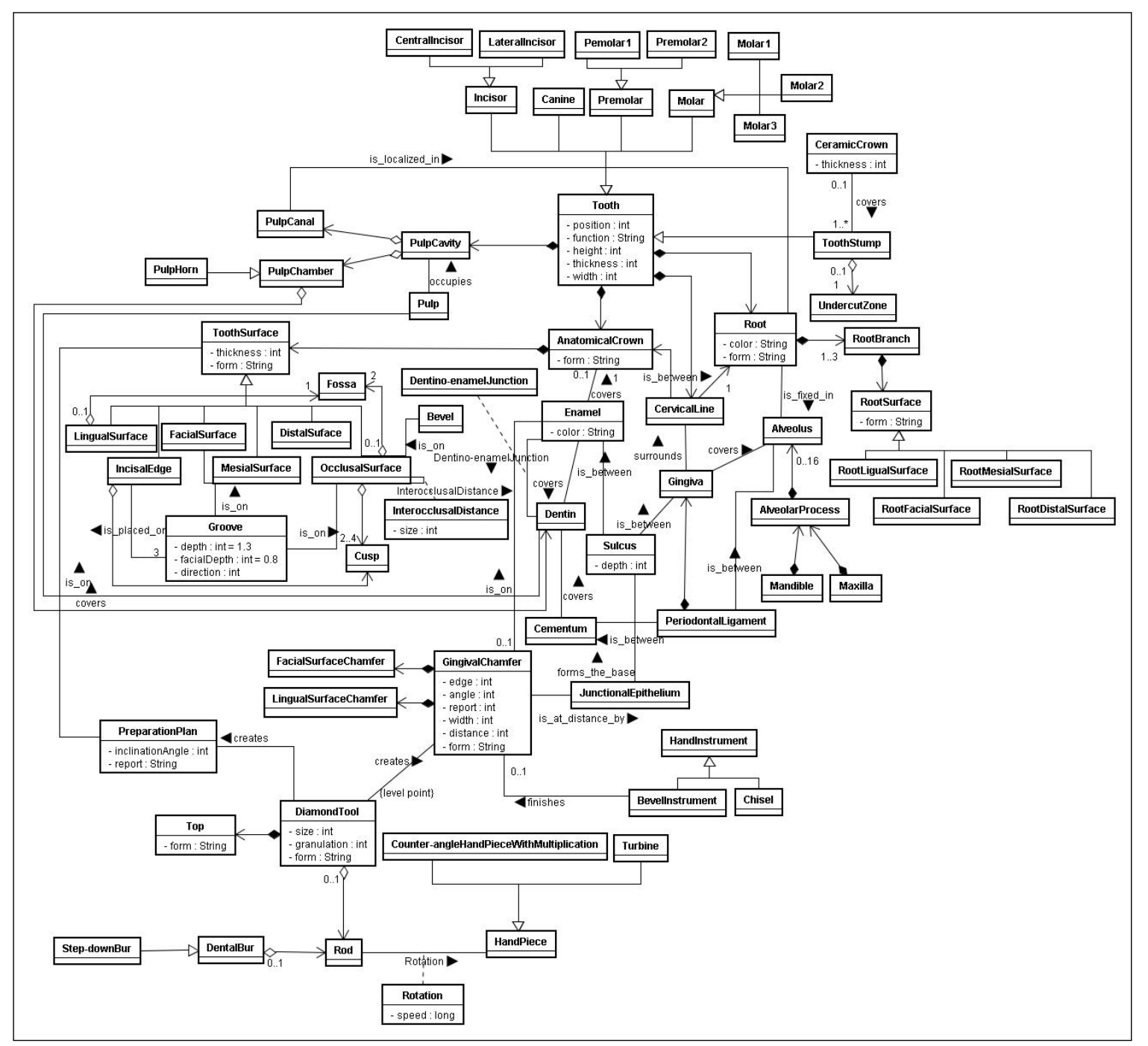

Figure 1: VirDenT domain model

To describe the system from the behavioral point of view we created the business use case diagram which form the business model business processes. The diagram contains eight business use cases that describe the preparation of teeth for all-ceramic crowns. One of the business 
use cases is "Central incisor preparation" whose UML business activity diagram is presented in Figure 2. The diagram contains activities that are part of the preparation techniques of the central incisors, their execution order and the business objects used as inputs or created or modified as outputs by the activities. Business objects are central incisors, grooves, incisal edges, and so on, and the tools used during execution of the activities, such as diamond tools and step-down burs.

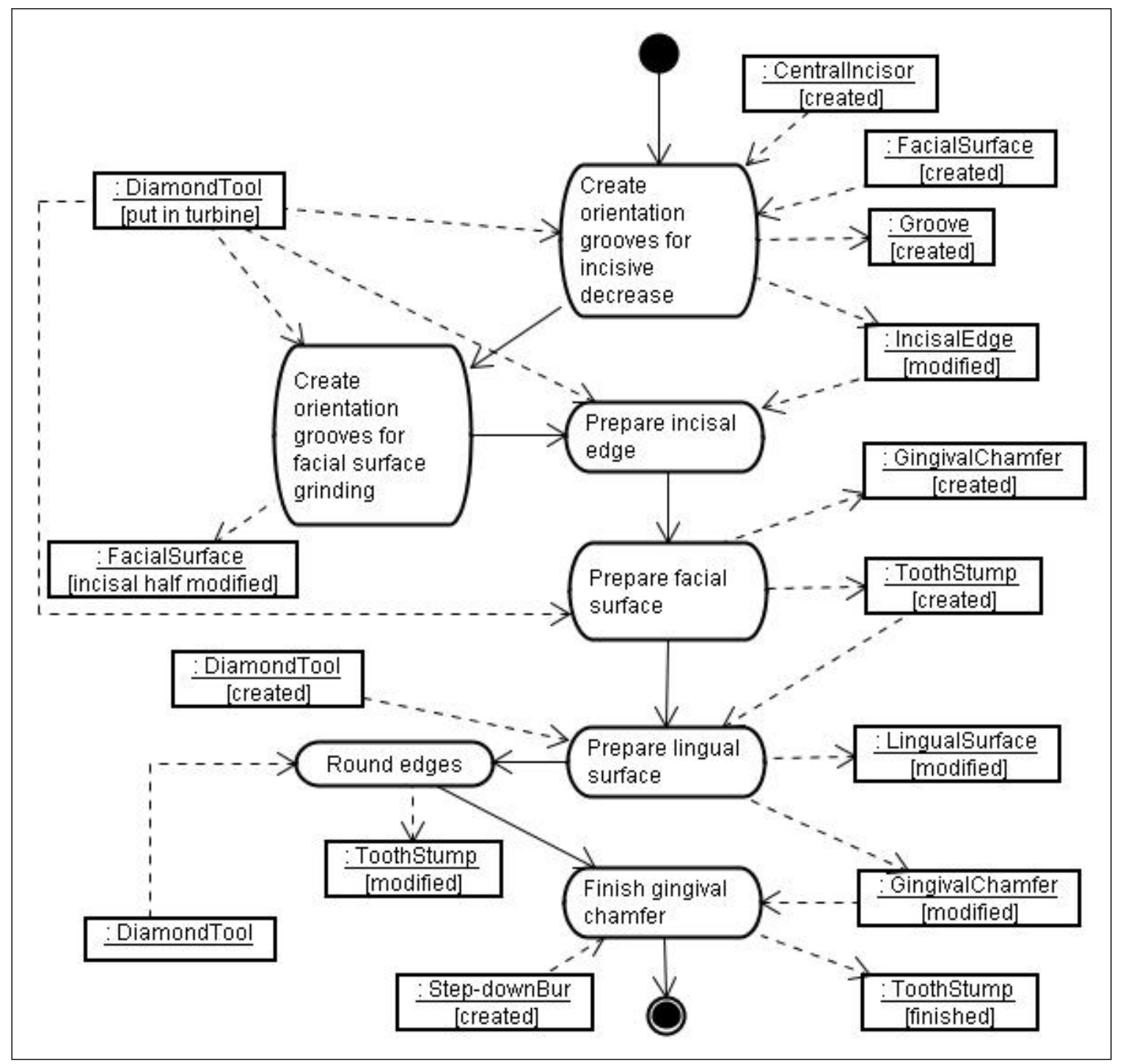

Figure 2: Business activity diagram of the VirDenT system

An ontology is a formal specification of the concepts intension and the intensional relationships that can exist between concepts. According to Guarino's definition, "an ontology is a logical theory accounting for the intended meaning of a formal vocabulary, i.e. its ontological commitment to a particular conceptualization of the world' [6]. In this paper, we present a domain ontology of the VirDenT system. 


\section{Method of construction of ontology}

The methodology of ontology construction is based on the few existent methodologies, like ontology development 101 [8], concern and business rule-oriented [1] and other ones. Our method performs a translation of the knowledge semantics described semi-formally in the UML models of the VirDenT information system, making sure in this way the ontology captures knowledge identified and described in the analysis of the information system. The method consists of the following steps: a) define the classes and the class hierarchy; b) define the relations of classes; c) ontologically describe the business rules; d) write additional constraints; e) verify the ontology. In addition, we mention that we used to build ontology language OWL DL (Web Ontology Language Description Logic) [16] and editor Protégé [5].

\section{VirDenT Ontology Construction}

Nowadays, there are some top-level ontologies (such as DOLCE, SUMO and BFO) which describe very general concepts like space, time, matter, object, event, etc., i.e. independent concepts by a particular domain or problem. Among these, we used the DOLCE ontology [7] and its modules such as D\&S [4], Temporal Relations, and so on. DOLCE is an ontology of particulars, in the sense that its domain of discourse is restricted to particulars. Other top-level ontologies might be used.

\subsection{Identify Classes and Creating Taxonomy}

First, classes are identified based on the UML class diagram of the model domain VirDenT system. So, each class in the class diagram has a corresponding OWL class, since an OWL class represents a set of individuals that form the extension of the concept mapped by class. Second, the most of the attributes of the classes have been transformed in physical, temporal and abstract qualities which are subclasses of the quality class of the DOLCE ontology. For example, Thickness and Form are two physical qualities, i.e. subclasses of the dolce:physical-quality class. Third, the activities of the business use cases are described ontologically as DOLCE processes, i.e. perdurants that fulfill the properties of cumulativity and homeomericity. For example, reduction is a process that executes during the "Create orientation grooves for incisive decrease" business activity (Figure 2). We classified reduction as a process, because the sum of two reductions is still a reduction occurrence and there are temporal parts of a reduction that are not reductions. These classes form the basis of the VirDenT ontology as during construction of taxonomy and ontology appear other new OWL classes.

The classes are organized in a taxonomy created on the basis of the subsumption relation. Two classes A and B are linked by subsumption relation if and only if every individual (instance) of the $\mathrm{B}$ class is also an individual of the A class [7]. In this case, we can say that A subsumes $\mathrm{B}$, or $\mathrm{A}$ is the superclass of the $\mathrm{B}$ class or B is a subclass of A. For example, the taxonomy of the amount of matters of the VirDenT ontology is presented in Figure 3.

\subsection{Defining the Conceptual Relationships}

Most of the conceptual relations of our ontology map the relations found in the DOLCE ontology and its sub modules, such as inherent-in, generic-constituent-of, participate-in, genericallydependent-on and so on. So, the qualities are linked by the concepts which they inhere in through the has-quality relation. For example, between the Enamel and Color concepts there is the has-quality relation (see Figure 3). 


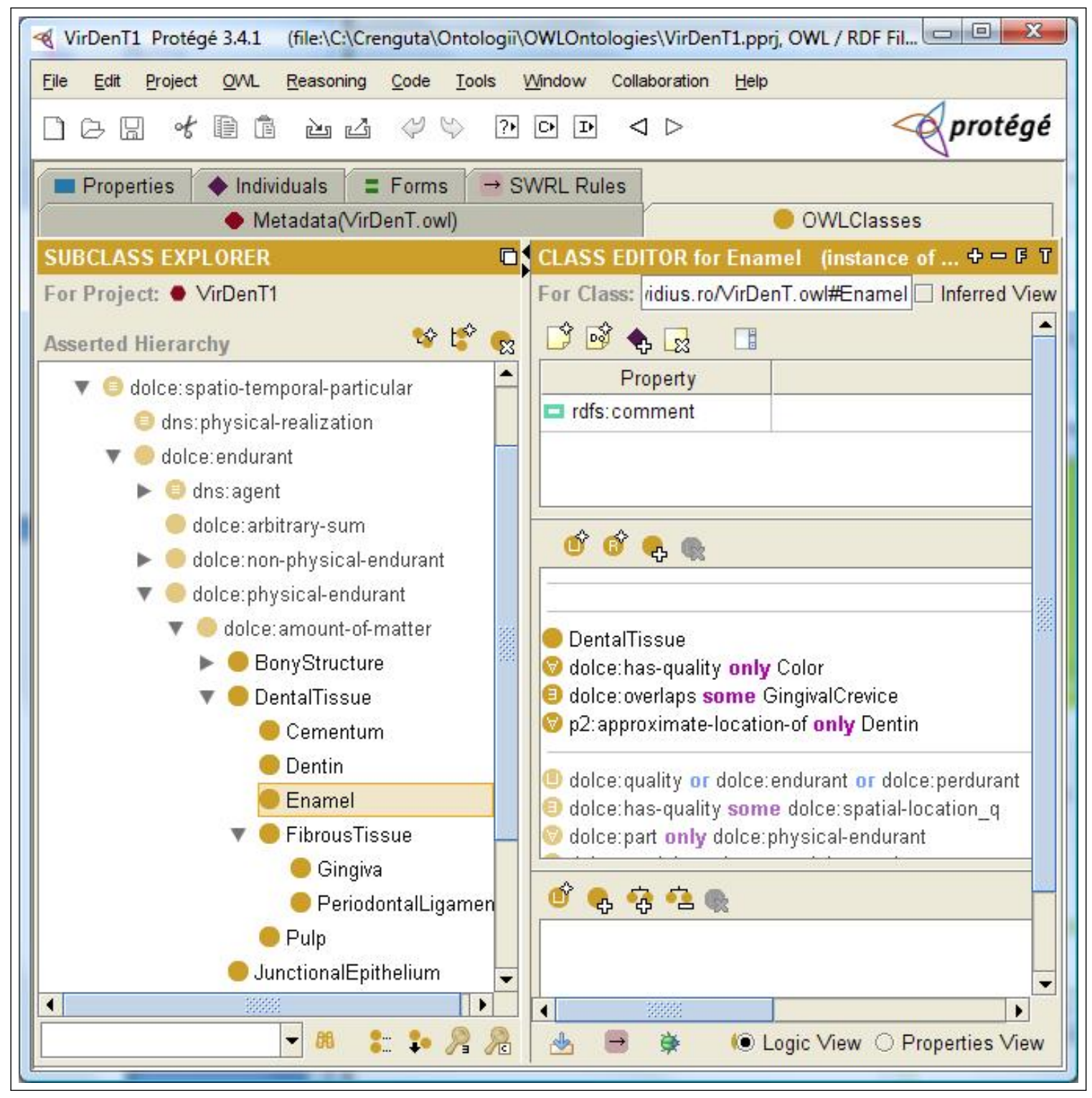

Figure 3: A subtaxonomy of our ontology

The aggregation relations from the UML class diagram (Figure 1) have been transformed into the temporary-component $\mathrm{D} \& \mathrm{~S}$ relation between the aggregate class and the class aggregated. For example, between the pair of concepts DiamondTool-Rod and DentalBur-Rod there is the temporary-component relation.

The composition relations from the class diagram (Figure 1) have been transformed into the specific-constant-constituent DOLCE relation which states that an entity consists of another entity for a period of time. For example, every individual of the AlveolarProcess concept is specific and constant constituent by individuals of the Alveolus concept.

Most of the associations of the UML class diagram (Figure 1) have correspondent imported ontological relations. For example, between the Enamel and Dentin concepts we have the approximate-location-of relation of the imported Spatial Relations ontology (Figure 3).

Observe in Figure 1 that each attribute has an UML data type, i.e. a classifier whose instances are values that attribute can take. Ontological modeling of data types of attributes is by datatype properties whose domains are the OWL classes associated with attributes and ranges are XML 
predefined data types. For example, the ThicknessValue is a datatype property that describes the data type of the thickness attribute of the Tooth class (Figure 1). The property domain is the Thickness class and range is the nonNegativeInteger predefined data type.

Furthermore, according to DOLCE ontology in any perdurant (process, state, etc.) at least one endurant participates in. For example, in the MakeGrooves process there are three participants: a groove, the incisal edge and a cylindrical-conical diamond tool with a flat top. So, any individual of the Groove concept participates in the MakeGrooves process for a period of time. This piece of knowledge is ontologically described introducing the DOLCE participant-in relation between the Groove and MakeGrooves concepts.

\subsection{Ontological Description of the Business Rules}

Business rules were identified during analysis of the VirDenT information system [2]. They describe knowledge that can be expressed ontologically using OWL DL or SWRL language. SWRL (Semantic Web Rule Language) allows us writing rules expressed in terms of OWL concepts: classes, properties and individuals [14]. For instance, the SWRL rule that expresses the piece of knowledge: "The incisal edge is decreased by $2 \mathrm{~mm}$ " is shown in the Figure 4.

Finally, we checked the ontology consistency with the help of the Protégé tool [5] version 3.4 and the Pellet reasoner system [11].

\section{Related Work}

There are few medical ontologies within dentistry. Park et al [10] created an OWL tooth ontology that embeds spatial relations. These relations describe the position of the tooth compared to other teeth. The relations derive from the formal relations in biomedical ontologies [12].

Pathogenic Pathway Database for Periodontitis was created based on an ontology that describes the molecular pathology of periodontal disease [13]. The database is used by programs which provide the following functionalities: displaying of the taxonomy in a tree-like view, key word search, showing causal relationships associated to a concept and a pathway browser.

SOMWeb is a semantic web-based system that provides IT support for clinicians and researchers in oral medicine to meet to review patient cases, establish a diagnosis, and decide on the most appropriate treatment plan for the patient. OWL ontologies are used in SOMWeb to represent oral medicine templates and knowledge, as well as to represent community models and data [3].

\section{Conclusions and Future Works}

We presented in this paper an ontology of the protocols for preparation of teeth for all-ceramic crowns. The ontology was developed based on the VirDenT information system.

Furthermore, we intend to use ontology to design the VirDenT software architecture and for the construction of a knowledge base that will form the persistence layer of the e-learning system.

\section{Acknowledgements}

We would like to thank Dr. C. Amariei, Dr. C. Ştefănescu, Dr. A. Zaharia and Dr. L. Petcu, professors at Faculty of Dental Medicine, Ovidius University of Constanta, Romania, for valuable comments to the study that forms the base of this paper. This research was supported by the Romanian Ministry of Education, Research and Innovation, National Centre of Programs Management, inside PNCDI-2 Partnerships program. 


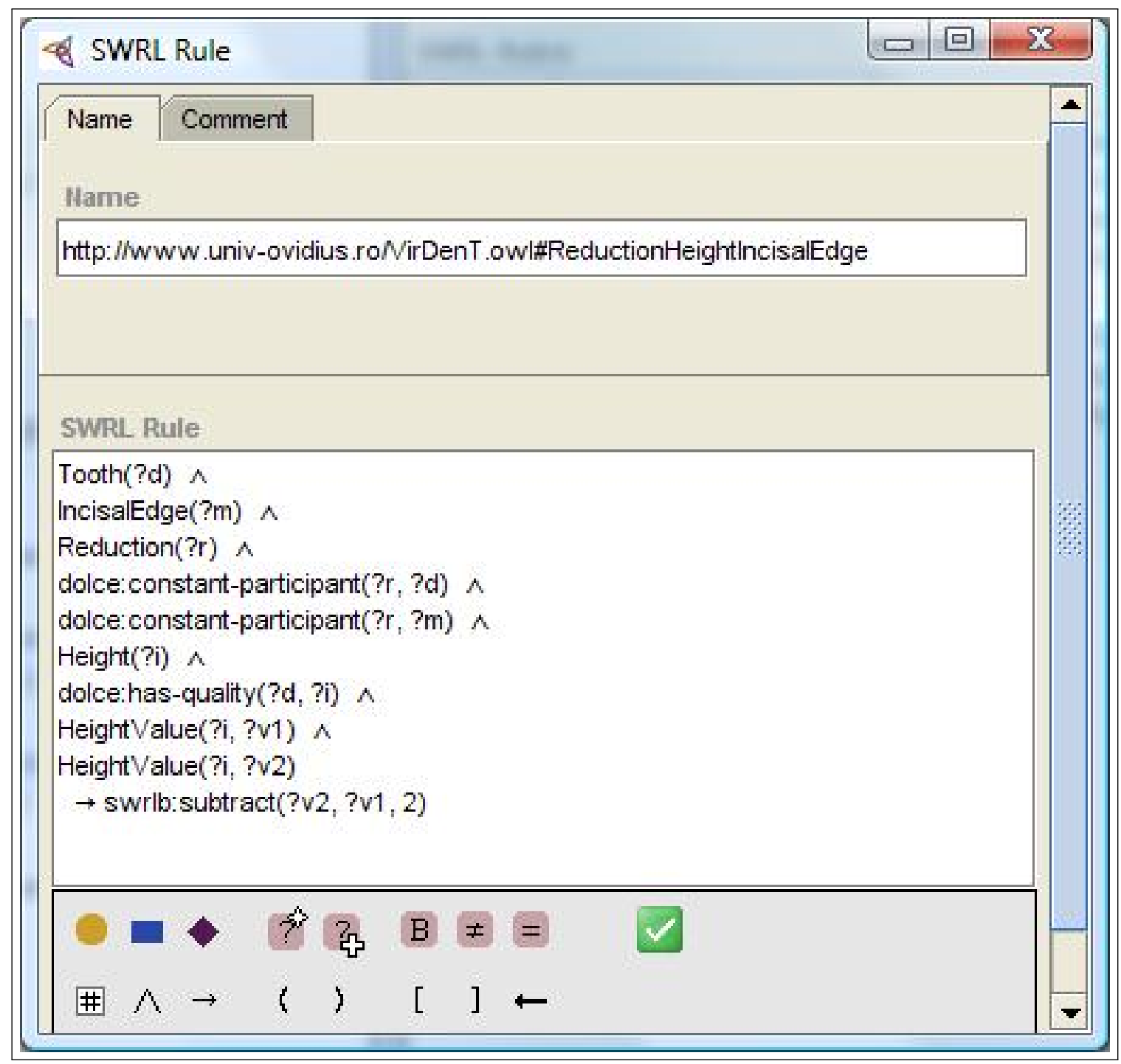

Figure 4: The SWRL rule of a piece of knowledge

\section{Bibliography}

[1] C. M. Bogdan, A. Păduraru, Concern and Business Rule-Oriented Approach to Construction of Domain Ontologies, International Journal of Computers, Communications and Control, Vol III (2008), Suppl. Issue: S, pp. 185-189.

[2] C. M. Bogdan, D. M. Popovici, Information System Analysis of an E-Learning System Used for Dental Restorations Simulation, submitted to Computer Methods and Programs in Biomedicine Journal, 2009.

[3] G. Falkman, M. Gustafsson, M. Jontell, O. Torgersson, SOMWeb: A Semantic Web-Based System for Supporting Collaboration of Distributed Medical Communities of Practice, Journal of Medical Internet Research, 10(3):e25, 2008.

[4] A. Gangemi, P. Mika, Understanding the Semantic Web through Descriptions and Situations. In Proceedings of the International Conference ODBASE03, Italy, Springer, pp. 689-706, 2003. 
[5] J. Gennari, M. Musen, R. Fergerson, W. Grosso, M. Crubzy, H. Eriksson, N. Noy, S. Tu, The evolution of Protégé-2000: An environment for knowledge-based systems development. International Journal of Human-Computer Studies, 58(1):89-123, 2003.

[6] N. Guarino, Formal Ontology and Information Systems. In Proceedings of FOIS'98, Trento, Italy, IOS Press, pp. 3-15, 1998.

[7] C. Masolo, S. Borgo, A. Gangemi, N. Guarino, A. Oltramari, WonderWeb Deliverable D18. Ontology Library. IST Project 2001-33052 WonderWeb: Ontology Infrastructure for the Semantic Web, 2003.

[8] N. F. Noy, D. McGuinness, A Guide to building ontologies: Ontology Development 101. A Guide to Creating Your First Ontology, March, 2001 at http://www.ksl.stanford.edu/people/dlm/papers/ontology101/ontology101-noymcguinness.html

[9] OMG, Unified Modeling Language Superstructure, version 2.0, ptc/03-0802, 2003.

[10] S. G. Park, H. G. Kim, M. K. Kim, Tooth Positional Ontology Represented in OWL, In Medinfo 200\%: Proceedings of the 12th World Congress on Health (Medical) Informatics; Building Sustainable Health Systems, pp. 2288-2289. Kuhn, Klaus A (Editor); Warren, James R (Editor); Leong, Tze-Yun (Editor). Amsterdam: IOS Press, 2007.

[11] Pellet Reasoner, http://clarkparsia.com/pellet

[12] B. Smith, W. Ceuster, B. Klagges, J. Kohler, A. Kumar, J. Lomax, C. Mungall, F. Neuhaus, A. Rector, C. Rosse, Relations in biomedical ontologies, Genome Biology, 6(5):R46, 2005.

[13] A. Suzuki, T. Takai-Igarashi, Y. Numabe, Hiroshi Tanaka, Development of a database and ontology for pathogenic pathways in periodontitis, In Silico Biology 9, 0020, 2009, pp. 233 244.

[14] SRWL W3C Submission, http://www.w3.org/Submission/2004/SUBM-SWRL-20040521/.

[15] B. Touati, P. Miara, D. Nathanson, Esthetic Dentistry and Ceramic Restorations, Martin Dunitz Ltd., London, 1999.

[16] World Wide Web Consortium. OWL Web Ontology Language Reference. W3C Recommendation, 2004. 